\title{
Hexagonal Honeycombs with Zero Poisson's Ratios and Enhanced Stiffness**
}

\author{
By Joseph N. Grima*, Ludovica Oliveri, Daphne Attard, Brian Ellul, Ruben Gatt, Gianluca Cicala and \\ Giuseppe Recca
}

In view of their potential applications in sandwich structures, there has been increasing interest in honeycomb networks. Several different types of honeycomb systems have been proposed each exhibiting different mechanical properties. Here we propose a new hexagonal honeycomb structure composed of two different geometrical features: a re-entrant feature which is known to generate auxetic behavior, and a non re-entrant feature found in regular hexagonal honeycombs which leads to conventional behavior. This results in a "semi re-entrant honeycomb" built of alternate conventional and auxetic layers. Finite element analysis and analytical modeling of these honeycombs show that they exhibit a zero Poisson ratio in one direction and a higher than normal Young's modulus in the orthogonal direction. We also show that by virtue of its zero Poisson's ratio, this honeycomb has a natural tendency to form cylindrical shaped curvatures, something which is very difficult to achieve with conventional or auxetic honeycombs.

In recent years there have been several studies on two-dimensional honeycombs, ${ }^{[1-24]}$ particularly honeycombs based on hexagonal cells as illustrated in Figure 1(a) in view of their simplicity and their utility in various practical applications ranging from aerodynamic components in cars and aircrafts to domestic internal doors. These include various studies aimed at studying their mechanical properties. In particular, in their seminal 1982 paper, ${ }^{[1]}$ Gibson and Ashby derived equations for the in-plane properties of hexagonal honeycomb systems such as the ones illustrated in Figure 1 deforming through flexure of the cell walls and show that the in-plane Poisson's ratios $v_{i j}$ and Young's moduli $E_{i}$ for loading in the $O x_{i}$ directions are given by:

[*] Prof. J. N. Grima, D. Attard, B. Ellul, R. Gatt Faculty of Science, Department. of Chemistry, University of Malta, Msida MSD 2080, Malta E-mail: joseph.grima@um.edu.mt; www: http://www.auxetic.info

Dr. L. Oliveri, Prof. G. Cicala, Dr. G. Recca

Faculty of Engineering, Department of Physical and Chemical, Methodologies for Engineering, University of Catania,

Viale A. Doria $n^{\circ}$ 6, 95125 Catania, Italy

[**] The financial support of the Malta Government Scholarship Scheme (Grant number ME 367/07/17) is gratefully acknowledged.

$$
\begin{aligned}
& E_{1}=E_{s}\left(\frac{t}{l}\right)^{3} \frac{\cos (\theta)}{[h / l+\sin (\theta)] \sin ^{2}(\theta)} \rightarrow E_{2}=E_{s}\left(\frac{t}{l}\right)^{3} \frac{h / l+\sin (\theta)}{\cos ^{2}(\theta)} \\
& v_{21}=v_{12}^{-1}=\frac{[h / l+\sin (\theta)] \sin (\theta)}{\cos ^{2}(\theta)}
\end{aligned}
$$

where as illustrated in Figure 1, $h$ is the length of the vertical ribs, $l$ the length of the inclined ribs, $t$ the thickness of the ribs, $\theta$ the angle that the inclined ribs make with the horizontal, taken to be positive for conventional honeycombs and negative for auxetic ones, and $E_{s}$ is the intrinsic Young's moduli of the honeycombs. These equations clearly suggest that honeycombs whose inclined cell walls are inverted inward commonly referred to as re-entrant honeycombs [i.e., honeycombs where $\theta$ as defined in Fig. 1 is negative, see Fig. 1(b)] can exhibit negative Poisson's ratios ${ }^{[1]}$ if they deform through flexure of the cell walls. Further studies on other honeycomb systems with a negative Poisson's ratio, chiral honeycombs ${ }^{[20-23]}$ and missing rib honeycombs ${ }^{[24]}$ in particular, have also been performed.

In this work we use finite elements modeling (FEM) and analytical modeling (AM) to analyze a novel class of hexagonal honeycomb structures which as illustrated in Figure 2 are constructed in such a way that their cells contain both re-entrant and non re-entrant features thus henceforth referred to as the "semi re-entrant" honeycomb. ${ }^{[25]}$ We show that these semi re-entrant systems exhibit two extremely useful mechanical properties namely zero Poisson's ratio for loading in the $O x_{1}$ direction and very high Young's moduli for loading in the $O x_{2}$ direction, the latter property being in 
a)

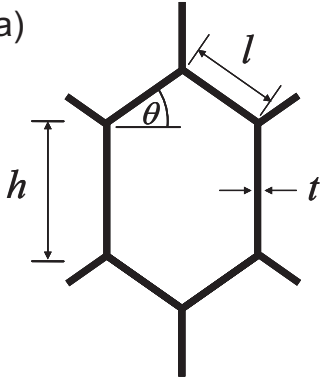

b)

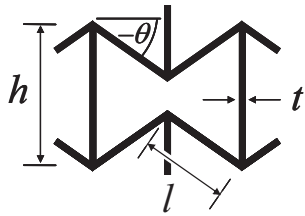

Fig. 1. The hexagonal honeycomb geometry considered by Gibson and Ashby. Shown here are (a) the conventional non re-entrant form and (b) the auxetic re-entrant form.

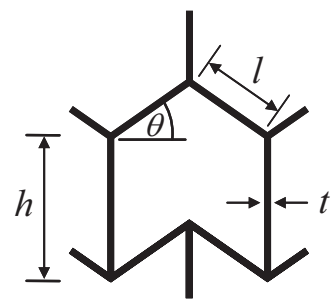

Fig. 2. The novel "semi re-entrant" hexagonal honeycomb geometry considered in this paper. Note that this honeycomb contains both re-entrant (normally associated with auxetic behavior) and non re-entrant (normally associated with conventional behavior) features.

accordance with the predictions made by $\operatorname{Lim}^{[26]}$ and Kocer et al. ${ }^{[27]}$ for composites made from alternate layers of auxetic and conventional materials.

\section{Simulations}

The mechanical behavior of various examples of the honeycombs illustrated in Figure 1 and 2, i.e., the conventional [Fig. 1(a)], the re-entrant [Fig. 1(b)] and the semi re-entrant honeycombs (Fig. 2) were simulated using the FEM software ANSYS in an attempt to understand how the new semi re-entrant configuration behaves when compared to the more traditional honeycombs. In particular simulations were performed using three values of the angle $\theta$ and three sets of $l / h$ ratios, namely $\theta=30,45$, and $60^{\circ}$ (i.e., $-30,-45$, and $-60^{\circ}$ in the case of the re-entrant systems) and with ( $l$, $h)=(100 \mathrm{~mm}, 180 \mathrm{~mm}),(100 \mathrm{~mm}, 250 \mathrm{~mm})$, and $(125 \mathrm{~mm}$, $250 \mathrm{~mm}$ ). All systems were modeled using the triangular 6-noded PLANE146 p-element which supports plane stress and plane strain analysis and free meshed in a manner which is fine enough such that convergence of the results was possible. In all systems $t$ was set at $2 \mathrm{~mm}$ and the material was modeled as perfectly elastic with a Young's modulus of $10 \mathrm{GPa}$ and a Poisson's ratio of 0.3 which is common for commercial plastics. Note that the dimensions $h$ and $l$ were measured along half the thickness of the ribs.

All of these systems were constructed as finite systems having $m \times n$ cells (see Fig. 3 for definition of $m$ and $n$ ) and were subjected to a $0.5 \%$ engineering strain in the $O x_{1}$ and $O x_{2}$ directions, respectively. In particular, in the case of loading in the $\mathrm{Ox}_{2}$ directions, the systems constructed had $(m \times n)=(5 \times 21)$ cells, where these values of $m$ and $n$ where

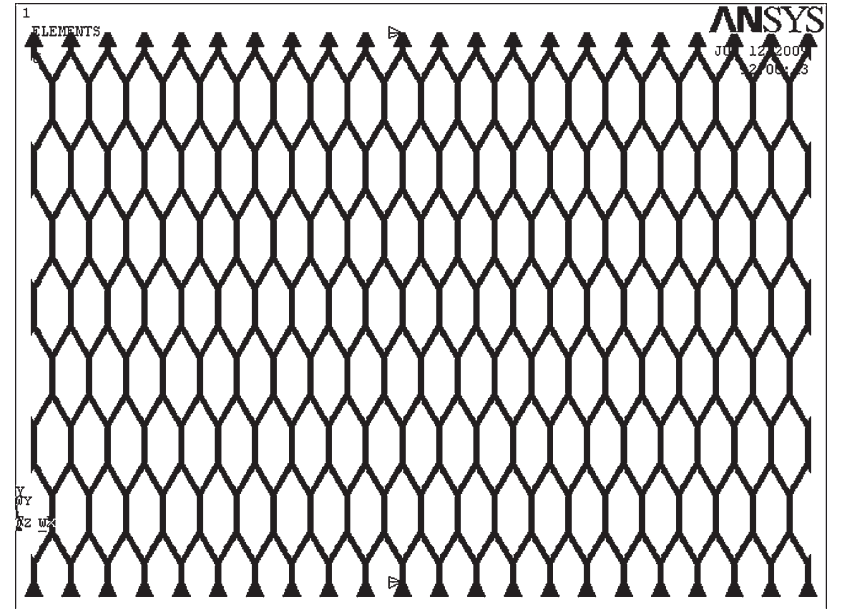

Fig. 3. Definition of the constraints used in ANSYS for modeling the behavior of the systems as they are stretched in the $O x_{2}$ dimension.

chosen because the aspect ratio of all the structures is bigger than 10 so as to fulfill the aspect ratio convergence limit suggested by the simulations of Kocer et al. for the "half auxetic-half conventional" semi re-entrant structures. The restraints were applied in such a way that all the nodes lying at the bottom face of the honeycomb were constrained to have a zero displacement in the $\mathrm{Ox}_{2}$ direction whilst all the nodes lying at the top face of the honeycomb were constrained to have a displacement in the $\mathrm{Ox}_{2}$ direction which corresponds to $0.5 \%$. In addition to this, the centremost nodes on both the top and bottom faces were also constrained to have a zero displacement in the $O x_{1}$ direction so as to ensure that there are no rigid body movements and also to ensure that the top and bottom faces remain aligned on top of each other. In the case of loading in the $O x_{1}$ directions, the systems constructed had $(m \times n)=(21 \times 5)$ cells with the restraints being applied in an equivalent manner.

Typical images of the deformed structures, where the displacements are scaled by a factor of 1 and $10 \%$ so as to aid interpretation, are shown in Figure 4. These images clearly show that with the exception of the semi re-entrant system when loaded in the $O x_{2}$ direction, all systems behaved in such a way that if one ignored the cells on the edges, the deformed structures may still be describable in terms of repeat units as all the non-edge cells in the structures deform in the same manner, i.e., behave as "well behaved" periodic structures. Furthermore, these well behaved structures (i.e., all systems with the exception of the semi re-entrant system when loaded in the $O x_{2}$ direction) were found to behave in such a way that their deformations are describable through flexure of the non-vertical cell walls in a manner that is conducive to positive Poisson's ratios in the case of the conventional honeycombs (stretching in both $O x_{1}$ and $O x_{2}$ ) and negative Poisson's ratios in the case of the fully re-entrant honeycombs (stretching in both $O x_{1}$ and $O x_{2}$ ). In the case of the re-entrant and non re-entrant honeycomb, these observations are in accordance with the assumptions made by Gibson and 


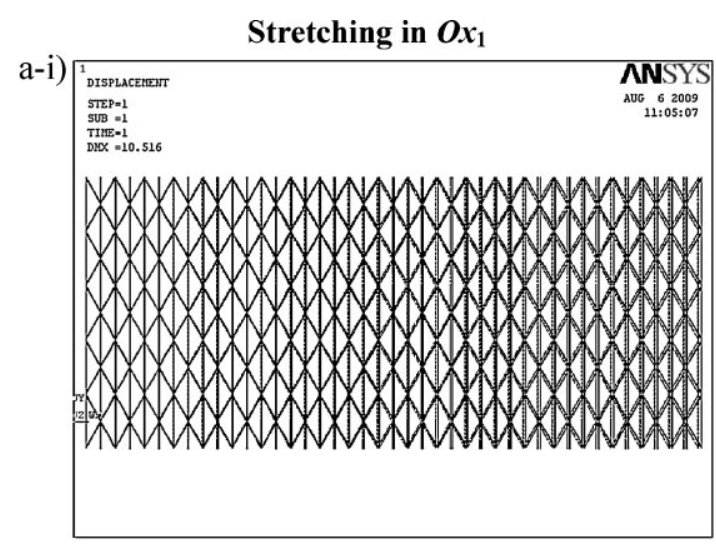

b-i)

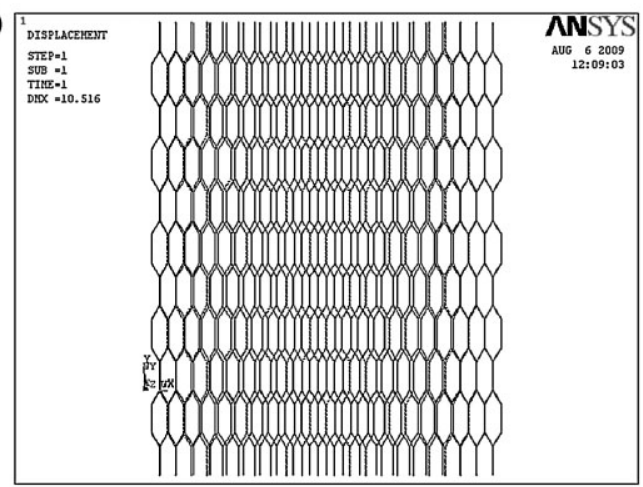

c-i)

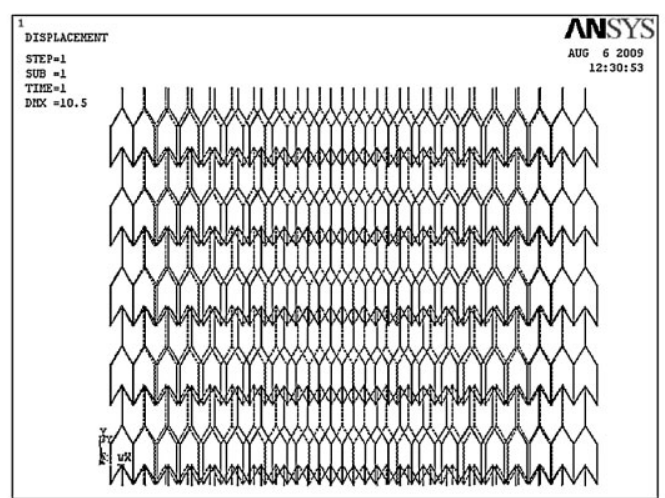

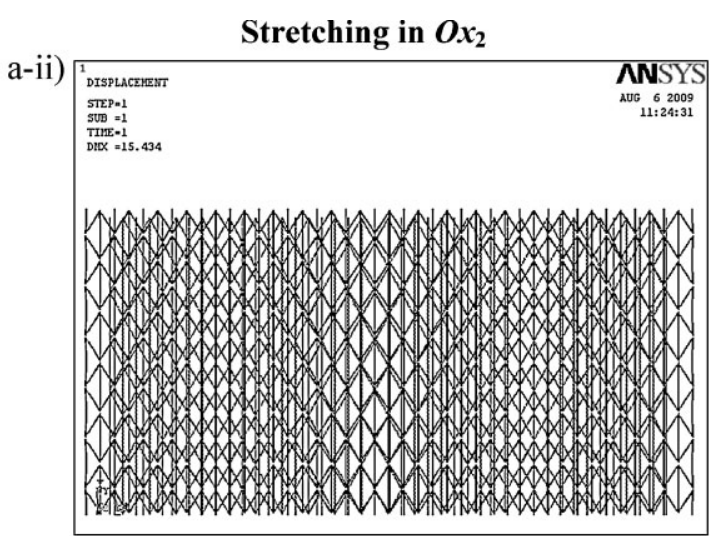

b-ii)

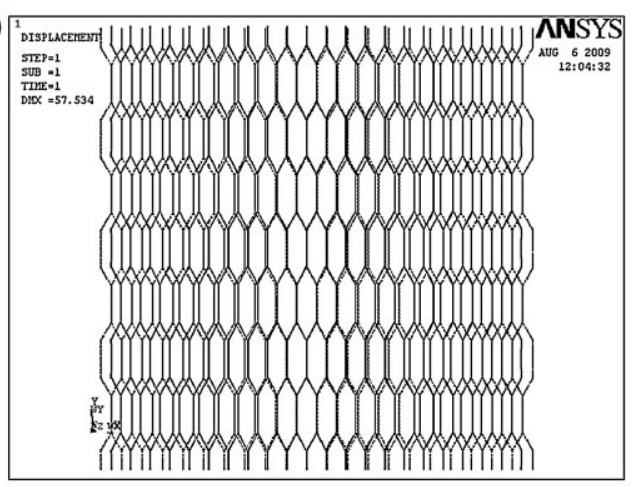

c-ii)

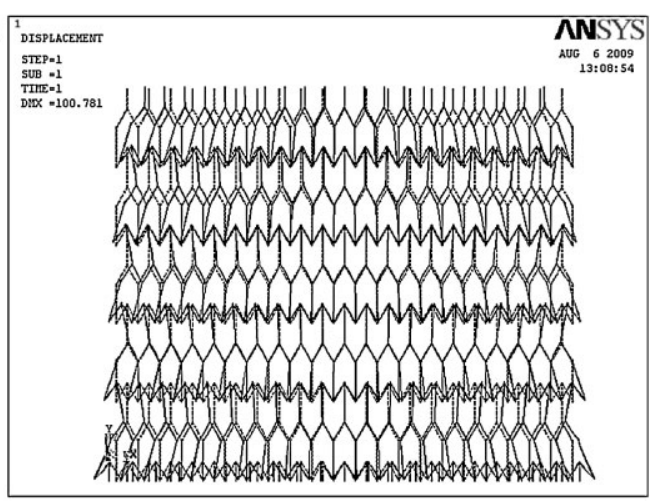

Fig. 4. Typical images showing the deformed (a) auxetic, (b) conventional, and (c) semi re-entrant systems for loading in the ( $i$ ) Ox $x_{1}$ and (ii) Ox $x_{2}$ directions. Shown here are the systems where $\theta, l, h=30^{\circ}, 100 \mathrm{~mm}, 180 \mathrm{~mm}$ respectively when they are subjected to a $0.5 \%$ displacement. Note that the deformations shown here have been magnified by a factor of $1 \%(a-i, b-i i, c-i i)$ and $10 \%(a-i i, b-i, c-i i)$.

Ashby $^{[1]}$ in their derivation of their analytical models for hexagonal honeycombs. These observations also suggest that analogous analytical models may be derived for the properties of the novel semi re-entrant honeycombs for stretching in the $O x_{1}$ direction using the unit-cell approach (see Section Analytical Modeling).

In contrast to all this, very different behavior was observed in the case of semi re-entrant systems when stretched in the $\mathrm{O} x_{2}$ direction where one may observe that the deformations of the cells are highly dependent on their position within the structure. This suggests that for loading in the $\mathrm{O} x_{2}$ direction, the semi re-entrant honeycombs may not be considered as "well behaved" periodic structures and thus the analytical expressions for their mechanical properties for stretching in the $O x_{2}$ direction may not be derived using the unit-cell approach.

In an attempt to further analyze the behavior of the structures and compare the simulated properties with the analytical models by Gibson and Ashby ${ }^{[1]}$ and those derived in this paper (Section Analytical Modeling), we also measured the Young's moduli and Poisson's ratios for the different honeycombs as simulated by FEM. In particular, the simulated Young's modulus $E_{2}$ for loading in the $\mathrm{Ox}_{2}$ direction which is defined as $E_{2}=\sigma_{2} / \varepsilon_{2}$ was calculated by measuring the total reactions $\sum F_{2}$ in the $O x_{2}$ direction of the fixed top nodes that result from the applied strain $\varepsilon_{2}=0.005$ 
and dividing this by $A_{2}$, the total dimension of top face of the honeycomb so as to obtain the resultant stress $\sigma_{2}$ in the $O x_{2}$ direction. The simulated Young's modulus $E_{1}$ for loading in the $O x_{1}$ was similarly calculated by measuring the total reactions in the $O x_{1}$ direction.

Furthermore, for the well behaved structures, we also attempted to measure the simulated Poisson's ratio of typical repeat units of the systems. In particular, the FEM simulated Poisson's ratio $v_{i j}$ was measured for the centremost cells (in an attempt to minimize edge effects) from the strains $\varepsilon_{i}$ in the $O x_{i}$ directions calculated from measurements of the displacements of particular easily identifiable key-points, illustrated in Figure 5, which give approximate measurements of the changes in dimensions of the repeat unit of the structure.

\section{Analytical Modeling}

The honeycomb considered here can be described by the unit-cell shown in Figure 2. Using the parameters defined in the figure, the projections of the undeformed unit-cell can be given by:

$X_{1}=2 l \cos (\theta)$

$X_{2}=2 h$

If the Young's modulus $E_{s}$ of the material is high enough for deformation by stretching of the ribs to be negligible then, when the system is loaded by a stress $\sigma_{1}$ in the $O x_{1}$ direction, each rib experiences a force $F$ at its end (see Fig. 6) causing it to flex. Using standard beam theory, for a rib of length $l$ and thickness $t$, the deflection at one end of the rib relative to the other is given by:

$\delta=\frac{l^{3} F \sin (\theta)}{12 E_{s} I}$

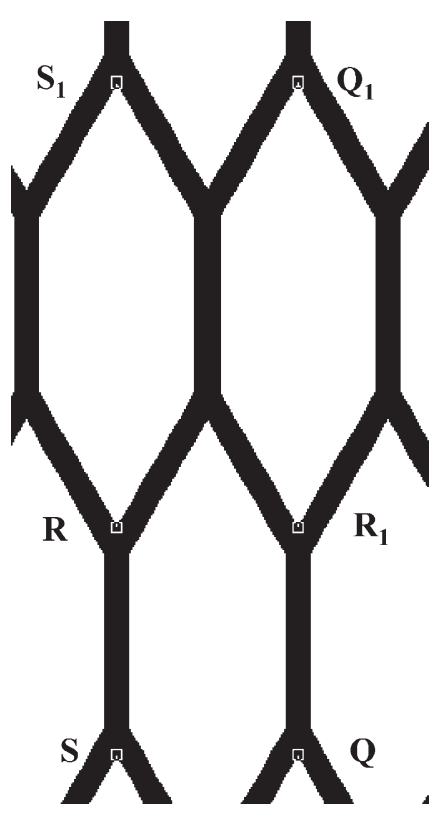

Fig. 5. Definition of the keypoints used to calculate strains
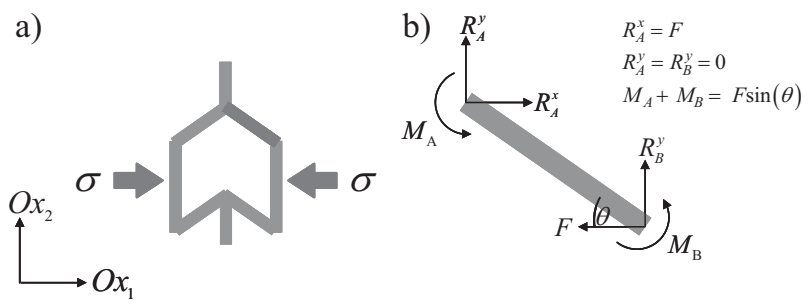

Fig. 6. (a) A unit-cell under a tensile stress and (b) deformation of the inclined ribs.

where $F$ is the force acting along the loading direction and $I=z t^{3} / 12$ is the second moment of inertia of the beam with $z$ being the out-of-plane thickness of the network. The force $F$ can be written in terms of the applied stress as $F=\sigma h z$ so that the total deflection $\delta$ perpendicular to the rib can be expressed as:

$\delta=\frac{\sigma h z l^{3} \sin (\theta)}{12 E_{s} I}$

and the total deflections $\delta_{O x_{1}}$ and $\delta_{O x_{2}}$ along the $O x_{1}$ and $O x_{2}$ directions are given by:

$\delta_{O x_{1}}=\frac{\sigma h z l^{3} \sin ^{2}(\theta)}{12 E_{s} I}$

and

$\delta_{O x_{2}}=\frac{\sigma h z l^{3} \sin (\theta) \cos (\theta)}{12 E_{s} I}$

Now it may be immediately noted that $\delta_{\mathrm{Ox}_{2}}$ for the re-entrant and conventional ribs is equal in magnitude but opposite in direction, i.e., the re-entrant ribs act to push the vertical ribs outward by $\delta_{\mathrm{Ox}_{2}}$ while the conventional ones act to pull it inward by $\delta_{O x_{2}}$ so that the net strain $\varepsilon_{2}$ along the $O x_{2}$ direction is zero, and therefore the Poisson's ratio $v_{12}$ can be simply given by:

$v_{12}=-\frac{\varepsilon_{2}}{\varepsilon_{1}}=0$

Note that this equation once again confirms that the Poisson's ratio is scale independent which means that this effect may be manifested at any scale ranging from the nano (molecular) level to the macro level.

Using Equations (1) and (5), the strain $\varepsilon_{1}$ along the $O x_{1}$ direction can be written as:

$\varepsilon_{1}=\frac{\delta_{O x_{1}}}{X_{1}}=\frac{\sigma h z l^{2} \sin ^{2}(\theta)}{12 E_{S} I \cos (\theta)}$

and using the standard definition of the Young's modulus, and substituting for $I$, it follows that:

$E_{1}=\frac{\sigma_{1}}{\varepsilon_{1}}=\left(\frac{t}{l}\right)^{3}\left(\frac{l}{h}\right) \frac{E_{s} \cos (\theta)}{\sin ^{2}(\theta)}$

This equation for the Young's modulus is similar to the equivalent equation derived by Gibson and Ashby for the re-entrant and non re-entrant honeycombs since both 
Table 1. Table showing the FEM results compared to those predicted by the analytical equations (AM) derived by Gibson and Ashby for the auxetic and conventional honeycombs and in this paper for the semi re-entrant honeycombs.

\begin{tabular}{|c|c|c|c|c|c|c|c|c|c|c|c|c|c|}
\hline \multirow[t]{3}{*}{$l, h(\mathrm{~mm})$} & \multirow[t]{3}{*}{$\theta$} & & \multicolumn{6}{|c|}{ Loading in $O x_{1}$ direction } & \multicolumn{5}{|c|}{ Loading in $\mathrm{O} x_{2}$ direction } \\
\hline & & & \multicolumn{3}{|c|}{$v_{12}$} & \multicolumn{3}{|c|}{$E_{1}(\mathrm{kPa})$} & \multicolumn{2}{|c|}{$v_{21}$} & \multicolumn{3}{|c|}{$E_{2}(\mathrm{kPa})$} \\
\hline & & & Auxetic & Conv. & $\begin{array}{c}\text { Semi } \\
\text { re-entrant }\end{array}$ & Auxetic & Conv. & $\begin{array}{l}\text { Semi } \\
\text { re-entrant }\end{array}$ & Auxetic & Conv. & Auxetic & Conv. & $\begin{array}{c}\text { Semi } \\
\text { re-entrant }\end{array}$ \\
\hline \multirow[t]{5}{*}{100,180} & \multirow[t]{2}{*}{$30^{\circ}$} & FEM & -1.153 & 0.651 & 0.000 & 224 & 122 & 159 & -0.864 & 1.530 & 163 & 312 & 1420 \\
\hline & & $\mathrm{AM}$ & -1.154 & 0.652 & & 213 & 120 & 154 & -0.867 & 1.533 & 160 & 283 & \\
\hline & \multirow[t]{2}{*}{$45^{\circ}$} & FEM & -0.647 & 0.282 & 0.000 & 113 & 46 & 66 & -1.538 & 3.532 & 260 & 629 & 4430 \\
\hline & & $\mathrm{AM}$ & -0.647 & 0.282 & & 104 & 45 & 63 & -1.546 & 3.546 & 247 & 567 & \\
\hline & \multirow[t]{2}{*}{$60^{\circ}$} & FEM & -0.309 & 0.108 & 0.000 & 68 & 21 & 33 & -3.203 & 9.165 & 676 & 1940 & 16500 \\
\hline \multirow[t]{6}{*}{100,250} & & $\mathrm{AM}$ & -0.309 & 0.108 & & 57 & 20 & 30 & -3.235 & 9.235 & 598 & 283 & \\
\hline & \multirow[t]{2}{*}{$30^{\circ}$} & FEM & -0.749 & 0.499 & 0.000 & 146 & 93 & 114 & -1.328 & 1.993 & 118 & 405 & 1070 \\
\hline & & $\mathrm{AM}$ & -0.750 & 0.500 & & 139 & 92 & 111 & -1.333 & 2.000 & 246 & 370 & \\
\hline & \multirow[t]{2}{*}{$45^{\circ}$} & FEM & -0.394 & 0.220 & 0.000 & 69 & 36 & 48 & -2.520 & 4.513 & 426 & 801 & 3210 \\
\hline & & $\mathrm{AM}$ & -0.394 & 0.220 & & 63 & 35 & 45 & -2.536 & 4.536 & 406 & 726 & \\
\hline & \multirow[t]{2}{*}{$60^{\circ}$} & FEM & -0.177 & 0.086 & 0.000 & 39 & 17 & 24 & -5.589 & 11.544 & 250 & 2440 & 12000 \\
\hline \multirow[t]{7}{*}{125,250} & & $\mathrm{AM}$ & -0.177 & 0.086 & & 33 & 16 & 21 & -5.660 & 11.660 & 1050 & 2150 & \\
\hline & \multirow{2}{*}{$30^{\circ}$} & FEM & -0.999 & 0.599 & 0.000 & 99 & 57 & 73 & -0.998 & 1.664 & 95 & 173 & 664 \\
\hline & & $\mathrm{AM}$ & -1.000 & 0.600 & & 95 & 57 & 71 & -1.000 & 1.667 & 95 & 158 & \\
\hline & \multirow[t]{2}{*}{$45^{\circ}$} & FEM & -0.547 & 0.261 & 0.000 & 48 & 22 & 30 & -1.822 & 3.818 & 155 & 346 & 2070 \\
\hline & & $\mathrm{AM}$ & -0.547 & 0.261 & & 45 & 21 & 29 & -1.828 & 3.828 & 150 & 314 & \\
\hline & \multirow[t]{2}{*}{$60^{\circ}$} & FEM & -0.255 & 0.101 & 0.000 & 28 & 10 & 15 & -3.902 & 9.876 & 407 & 106 & 7880 \\
\hline & & AM & -0.255 & 0.101 & & 24 & 10 & 14 & -3.928 & 9.928 & 372 & 939 & \\
\hline
\end{tabular}

equations suggest that the moduli tend to infinity as $\theta$ approaches $0^{\circ}$ and zero as $\theta$ approaches $90^{\circ}$. There are also similarties in the way the Poisson's ratio varies with $t, h$, and $l$.

\section{Results and Discussion}

The simulated Young's moduli and Poisson's ratio for the various structures are shown in Table 1 where they are compared to the equivalent properties as predicted by the analytical expressions (when available). In the case of the conventional and re-entrant honeycombs, these measurements of the Poisson's ratio and Young's moduli clearly show that the results of the simulations are in excellent agreement with the predictions made by Gibson and Ashby. This is particularly true for the Poisson's ratio where the difference between the FEM data and the predicted values from the equations was always less than $1 \%$. This is very important as it validates the FEM simulation methodology employed in this paper.

In the case of the novel semi re-entrant honeycombs, the measurements of the Poisson's and Young's moduli for stretching in the $O x_{1}$ direction were also found to be in excellent agreement with the analytical predictions made in this paper and confirm the zero Poisson's ratios for all conformations of the honeycombs when the system is loaded in the $O x_{1}$ direction. This result is of considerable practical significance in view of the fact that systems with zero Poisson's ratio are useful in various practical applications ranging from substitutes to naturally occurring cork which also exhibits zero Poisson's ratio to the manufacture of smart tubes which have variable diameter without changing the length of the tube. This is due to the fact that if such honeycombs are formed in the shape of cylinders (tubes) with the $O x_{2}$ direction corresponding to the height of the cylinder, then the zero Poisson's ratio for stretching in the $O x_{1}$ direction will give the cylinder the ability to increase or decrease in diameter without changing the height.

In the case of loading in the $O x_{2}$ direction, we note that the simulated moduli $E_{2}$ of the semi re-entrant honeycombs are always significantly larger than the moduli of the more traditional honeycombs where the increment in the Young's modulus is dependent on the geometry of the system. In fact, as illustrated in Figure 7, we note that for any particular $\theta, h$, and $l$ combination used in our simulations, the simulated moduli in the $O x_{2}$ direction for the semi re-entrant, $E_{2}^{\mathrm{SR}}$, is up to a factor of 8.5 greater than $E_{2}^{\mathrm{a} \text {-c-max }}$ which we define as the maximum from $E_{2}$ of the auxetic re-entrant honeycomb and $E_{2}$ of the conventional non re-entrant honeycomb having the same values of $\theta, h$, and $l$ and up to 16.5 times greater than $E_{2}^{\mathrm{a}-c-m i x}$ which we define as the value of $E_{2}$ that would have been obtained if the rule of mixtures had to be applied to $E_{2}$ of the re-entrant honeycomb and $E_{2}$ of non re-entrant honeycomb having the same values of $\theta, h$, and $l$. Note that bigger enhancements are expected if other $\theta, h$, and $l$ combinations are also used. Once again, this finding that such honeycombs exhibit high values of the Young's moduli is of obvious considerable practical significance. ${ }^{[26,27]}$

We also analyzed the extent of increase of the Young's modulus in the $O x_{2}$ direction as a function of $\Delta v_{21}$, the difference in the Poisson's ratio for loading in the $\mathrm{Ox}_{2}$ direction between the fully conventional and the fully auxetic re-entrant honeycombs. As illustrated in Figure 8, we found that for any particular $h / l$ combination, the extent of increase of the Young's modulus increases as the difference in the 
$(h, l)=\diamond(180 \mathrm{~mm}, 100 \mathrm{~mm}) \quad \triangle(250 \mathrm{~mm}, 125 \mathrm{~mm}) \quad \square(250 \mathrm{~mm}, 100 \mathrm{~mm})$
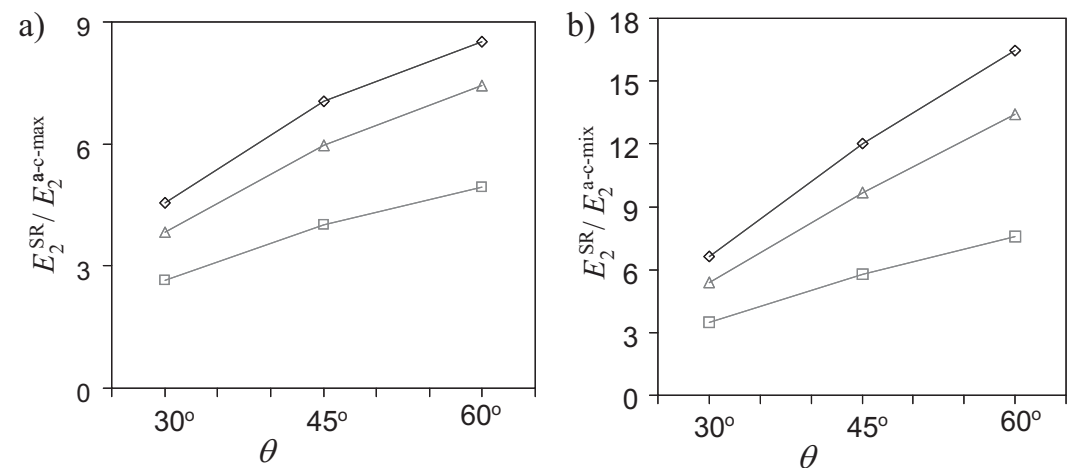

Fig. 7. The enhancement of the Young's moduli in the of the semi re-entrant honeycomb plotted against q where (a) shows the enhancement over the maximum modulus of the equivalent re-entrant and non re-entrant honeycombs and (b) shows the enhancement over the modulus that would have been obtained by applying the rule of mixtures to moduli of the equivalent re-entrant and non re-entrant honeycombs. Note that the values of the moduli used in this plot are those generated by the FEM simulations.

Poisson's ratio between the fully conventional and the fully auxetic re-entrant honeycombs increases. This result conforms with the predictions made by Kocer et al. who studied a class of hypothetical composite materials made from one layer of auxetic isotropic material and one layer of conventional isotropic materials which was shown through FE modeling to exhibit Young's moduli in the direction orthogonal to that of the layers which were significantly higher than that predicted by the rule of mixtures. In fact, in their simulations, Kocer et al. note that the highest enhancement in the Young's modulus is shown when the differences in the Poisson's ratio approaches that of 1.5, the limit imposed by the thermodynamics limits that the Poisson's ratios of isotropic materials must range within the bounds $-1 \leq v \leq+0.5$. We note that the Poisson's ratios of the component honeycombs presented here are not bound by these strict limits since such honeycombs are free to adopt any Poisson's ratio values ranging from $-\infty$ (a limit

\section{$(h, l)=\diamond(180 \mathrm{~mm}, 100 \mathrm{~mm}) \quad \Delta(250 \mathrm{~mm}, 125 \mathrm{~mm}) \quad \square(250 \mathrm{~mm}, 100 \mathrm{~mm})$}
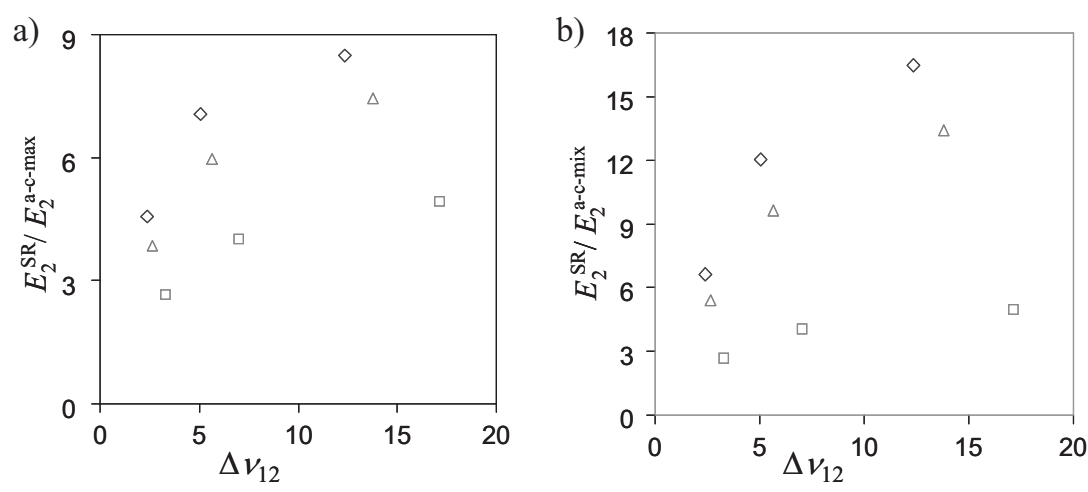

Fig. 8. The enhancement of the Young's moduli in the semi re-entrant honeycomb plotted against $\Delta v_{21}$ where (a) shows the enhancement over the maximum modulus of the equivalent re-entrant and non re-entrant honeycombs and (b) shows the enhancement over the modulus that would have been obtained by applying the rule of mixtures to moduli of the equivalent re-entrant and non re-entrant honeycombs. Note that the values of the moduli and Poisson's ratio used in this plot are those generated by the FEM simulations. approached by the re-entrant honeycomb when $-\theta \rightarrow-90^{\circ}$ ) to $+\infty$ (a limit approached by the conventional honeycomb when $\theta \rightarrow 90^{\circ}$ ). In fact, the equations for the moduli as derived by Gibson and Ashby ${ }^{[1]}$ may be used to derive an equation for the difference in the Poisson's ratio in the $O x_{2}$ directions between the fully auxetic and the conventional honeycombs which is given by:

$\Delta v_{21}=\frac{2 h \sin (\theta)}{l \cos ^{2}(\theta)}$

an expression which may take values ranging from zero (as $\theta \rightarrow 0^{\circ}$ ) to $+\infty$ (as $\theta \rightarrow 90^{\circ}$ ). This equation also suggests that for any particular value of $\theta$, the actual value of $\Delta \nu_{21}$ may be fine tuned through careful choice of the $h / l$ ratio where higher differences of the Poisson's ratios may be obtained for higher $h / l$ ratios.

However, it should be emphasized that as clearly illustrated in Figure 8, the relationship between $\Delta v_{21}$ and the extent of increase in the Young's moduli $E_{2}$ for the structures presented here is not a simple one and the ratio $h / l$ also seems to play an important role in determining the extent of increase. In fact, as illustrated in Figure 8, our simulations suggest that for particular values of $\Delta v_{21}$, the maximum enhancements are obtained for lower $h / l$ ratios, i.e., systems which have short vertical ribs. This may be explained through analysis of the deformed structures following stretching in $O x_{2}$. As Figure 4 clearly illustrates, strains in the $O x_{2}$ direction need to be accompanied by flexure of the vertical ribs which can occur more easily in systems with longer (and more slender) vertical ribs. Such flexure is required to accommodate the changes in the horizontal dimensions between layers having the "re-entrant" set of inclined ribs which expand laterally in the $O x_{1}$ when the honeycomb is stretched in $O x_{2}$ and the "non re-entrant" set of inclined ribs which shrinks laterally in the $O x_{1}$ when the honeycomb is stretched in $O x_{2}$. In other words, the net Young's modulus in the $O x_{2}$ direction increases as the extent of flexure of the vertical ribs decreases, something which may be achieved by using low $h / l$ ratios. However, given the fact that the factor $h / l$ is also involved in the term $\Delta v_{21}$ where, from the aspect of $\Delta v_{21}$ alone, higher $h / l$ result in larger $\Delta \nu_{21}$ values which in turn result in higher enhancements of the net Young's modulus in the $\mathrm{O} x_{2}$ direction, one should seek to optimize the values of the $h / l$ ratios in such a way to obtain maximum stiffness enhancements. In this respect, from the range of structures reported in this paper we note that if the analysis had to be performed against the geometric parameters $\theta$ and $h / l$, we clearly note that providing that the honeycombs are 

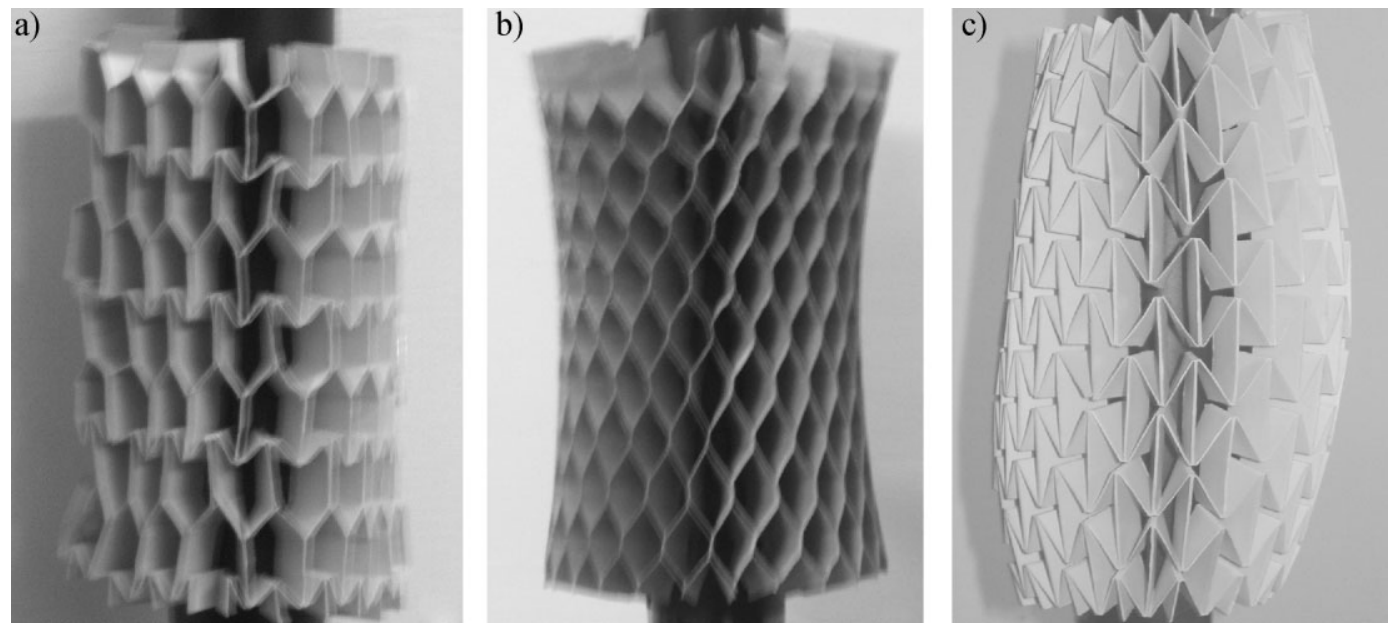

Fig. 9. Images of "tubes" formed from (a) a semi re-entrant honeycomb; (b) a conventional honeycomb and (c) a re-entrant auxetic honeycomb. Note that only the semi re-entrant honeycomb can be easily shaped into a tube since the conventional honeycomb tends to assume a "concave" shape whilst the auxetic honeycomb tends to assume a "convex" shape.

within the range of $\theta$ and $h / l$ analyzed here, maximum enhancements of the Young's moduli when stretching the semi re-entrant honeycombs in the $O x_{2}$ directions (as compared to stretching of the conventional/re-entrant honeycombs), occurs for larger values of $\theta$ and higher $h / l$ ratios.

Before we conclude, we would like to highlight that in addition to the obvious use of such honeycombs in applications which would require no change in the lateral direction when the honeycomb is uniaxially stretched or compressed, by having a zero Poisson's ratio, the honeycomb presented here, which has a thickness $Z$ in the third dimension, has the ability to form cylindrical shaped surface (tubes), something which as illustrated in Figure 9, neither the conventional nor the auxetic re-entrant equivalent can do. (Conventional honeycombs tend to form saddle shaped surfaces and auxetic re-entrant honeycombs tend to form dome shaped surfaces.) The reason for this is that if one tries to form a cylindrical shape or a tube from a conventional honeycomb sheet of dimensions $X \times Y \times Z$ in such a way that $Y$ will become the length of the tube and $X$ will become the neutral axis along the circumference of the tube, then one will note that the circumference on the inner side of the tube is $2 \pi Z$ shorter than the outer circumference. This puts the outer surface under radial tension and the inner surface under radial compression. As a consequence of the positive Poisson's ratio, the outer surface contracts along the longitudinal direction, while the inner one expands forcing the cylinder to adopt a curve in the opposite direction to the direction of bending, thereby causing the tube to appear with a "concave" shape as illustrated in Figure 9(b). Similarly, a tube made from an auxetic honeycomb will appear "convex" as illustrated in Figure 9(c). However, such differences between the inner and outer lengths will not appear if the honeycomb has a zero Poisson's ratio with the result that such honeycombs (or materials having a zero Poisson's ratio) are fully amenable to form cylinders or tubes.
This feature is particularly useful for honeycombs which have to be used as the core of sandwich composites for use in applications where the sandwich will be morphed into cylindrical shapes.

\section{Conclusions}

In this paper we have presented a novel type of honeycomb which can be considered as a semi re-entrant composed from elements pertaining to the more traditional conventional and auxetic hexagonal honeycombs (i.e., both re-entrant and non re-entrant features) which exhibit three extremely useful mechanical properties namely:

- zero Poisson's ratio for loading in the $O x_{1}$ direction, a property which is found to be scale independent, i.e., manifestable at any scale of structure ranging from the nano (molecular) level to the macroscale;

- very high Young's moduli for loading in the $O x_{2}$ direction when compared to the traditional re-entrant and non re-entrant honeycombs; and

- a natural tendency to form cylindrical or tubular shaped objects.

Given these benefits, we envisage that this work will stimulate further research into these types of honeycombs with the hope that the models presented here may form the basis for the synthesis and or manufacture of such honeycombs exhibiting the properties predicted here.

Received: April 15, 2010 Final Version: May 27, 2010

[1] L. J. Gibson, M. F. Ashby, G. S. Schajer, C. I. Robertson, Proc. R. Soc. Lond. A 1982, 382, 25.

[2] I. G. Masters, K. E. Evans, Compos. Struct. 1996, 35, 401. 
[3] K. E. Evans, A. Alderson, F. R. Christian, J. Chem. Soc. Faraday Trans. 1995, 91, 2671.

[4] M. A. Nkansah, K. E. Evans, I. J. Hutchinson, Modell. Simul. Mater. Sci. Eng. 1994, 2, 337.

[5] F. Scarpa, P. Panayiotou, G. Tomlinson, J. Strain Anal. 2000, 35, 383.

[6] A. Bezazi, F. Scarpa, C. Remillat, Compos. Struct. 2005, 71, 356.

[7] H. X. Zhu, N. J. Mills, Int. J. Solids Struct. 2000, 37, 1931.

[8] E. Wu, W. S. Jiang, Int. J. Impact Eng. 1997, 19, 439.

[9] T. Wierzbicki, Int. J. Impact. Eng. 1983, 1, 157.

[10] S. D. Papka, S. Kyriakides, Acta Mater. 1998, 46, 2765.

[11] S. D. Papka, S. Kyriakides, Int. J. Solids Struct. 1999, 36, 4367.

[12] S. D. Papka, S. Kyriakides, Int. J. Solids Struct. 1999, 36, 4397.

[13] F. Scarpa, P. J. Tomlin, Fatigue Fract. Eng. Mater. Struct. 2000, 23, 717.

[14] F. C. Smith, F. Scarpa, B. Chambers, IEEE Microw. Guided W. 2000, 10, 451.

[15] M. Ruzzene, L. Mazzarella, T. Panagiotis, F. Scarpa, J. Intel. Mater. Syst. Struct. 2002, 13, 587.
[16] M. J. Silva, L. J. Gibson, W. C. Hayes, Int. J. Mech. Sci. 1995, 37, 1161.

[17] M. A. Fortes, M. F. Ashby, Acta Mater. 1999, 47, 3469.

[18] J. P. M. Whitty, A. Alderson, P. Myler, B. Kandola, Compos. Part A - Appl. S. 2003, 34, 525.

[19] J. Rasburn, P. G. Mullarkey, K. E. Evans, A. Alderson, S. Ameer-Beg, W. Perrie, AIChE J. 2001, 47, 2623.

[20] D. Prall, R. S. Lakes, Int. J. Mech. Sci. 1997, 39, 305.

[21] J. Martin, J. J. Heyder-Bruckner, C. Remillat, F. Scarpa, K. Potter, M. Ruzzene, Phys. Status Solidi B 2008, 245, 570.

[22] F. Scarpa, S. Blain, T. Lew, D. Perrott, M. Ruzzene, J. R. Yates, Compos. Part A - Appl. S. 2007, 38, 280.

[23] J. N. Grima, R. Gatt, P. S. Farrugia, Phys. Status Solidi B 2008, 245, 511.

[24] N. Gaspar, X. J. Ren, C. W. Smith, J. N. Grima, K. E. Evans, Acta Mater. 2005, 53, 2439.

[25] J. N. Grima, L. Oliveri, D. Attard, B. Ellul, R. Gatt, G. Cicala, G. Recca, Paper presented at the 6th International Workshop on Auxetics \& Related Systems, Bolton (UK), September 2009.

[26] T. C. Lim, Eur. J. Mech. A - Solid 2009, 28, 752.

[27] C. Kocer, D. R. McKenzie, M. M. Bilek, Mater. Sci. Eng. A 2009, 505, 111. 